\title{
Combined ANN/ EVOP Factorial Design Approach for Media Screening for Cost-effective Production of Alkaline Proteases from Rhizopus oryzae (SN5) / NCIM-1447 under SSF
}

\author{
Sangeeta Negi ( $\sim$ sn5@mnnit.ac.in ) \\ Motilal Nehru National Institute of Technology https://orcid.org/0000-0002-9974-9171 \\ Kumari Vibha \\ Motilal Nehru National Institute of Technology
}

Original article

Keywords: Rhizopus oryzae, EVOP, Artificial Neural Networks (ANN), alkaline protease, wheat bran

Posted Date: January 30th, 2020

DOl: https://doi.org/10.21203/rs.2.22318/v1

License: (c) (1) This work is licensed under a Creative Commons Attribution 4.0 International License.

Read Full License

Version of Record: A version of this preprint was published at AMB Express on March 27th, 2020. See the published version at https://doi.org/10.1186/s13568-020-00996-7. 


\section{Abstract}

In order to achieve high yield of fungal protease in a very cost effective way and to meet its increased market demand, current study deals with the screening of various agro-wastes as carbon source for the production of protease from Rhizopus oryzae (SN5) / NCIM-1447 under solid state fermentation. Substrates and culture parameters such as wheat bran, soybean meal, black-gram husk, rice husk, mixture of wheat bran, soybean meal, nitrogen sources, $\mathrm{pH}$, temperature and incubation time were first optimized with one factor at time strategy and then EVOP factorial and yield of alkaline protease was achieved $412.8 \mathrm{U} / \mathrm{gds}$ at $28 \mathrm{oC}$ and $\mathrm{pH}=6$ after 72 hours of fermentation taking wheat bran and soybean as a substrate in 4:1 ratio. Further Artificial Neural Networks (ANN), was trained with data of EVOP and yield of protease was enhanced up to $422.6 \mathrm{U} / \mathrm{gds}$ with wheat bran: soyabean in ratio of 70:30, $\mathrm{pH} 6.2$ at $30^{\circ} \mathrm{C}$. The evolved process and Rhizopus oryzae (SN5)/NCIM-1447 strain would be promising for protease production at industrial scale at low cost.

\section{Introduction}

Proteases or peptidyl-peptide hydrolases (EC: 3.4.21-24 and 99) catalyze the total hydrolysis of proteins by acting on their peptide bonds. They are able to catalyse both hydrolytic and synthetic reactions, based on substrate, $\mathrm{pH}$ and other physicochemical conditions. Alkaline proteases occupy a pivotal position with respect to their applications in both physiological and commercial fields. They are mainly used in food industry as probiotics, in food processing, in beverages etc, as antimicrobial agent in medicine, textile manufacturing, detergent, food-processing, paper and pulp industries, leather industry etc.

Proteases posses all functions, such as high microbial activity, can make protecting layer over intestinal wall against pathogens and can also modulate host immune system, which makes them best food supplements for all purposes [1, 2]. They are the part of various important biological functions i.e., cardiovascular, nervous, gastrointestinal and immune systems etc., therefore used as medicine through functional food also.

The future of protease enzymes in the pharmaceutical industry shows promising growth in forthcoming years. The global protease enzymes market is envisaged to grow at a healthy CAGR of 5.3\%. From 2014 to 2019 , protease consumption in China is expected to elevate at a CAGR of $6 \%$, reaching upto USD 272.6 million by 2019 (Global Trends \& Forecasts to 2019). In order to satiate the increasing demand, it is extremely necessary to formulate cost-effective methods for production of highly active and stable proteases.

Proteases sources are animals, plants and microorganisms. But on the basis of various economical, technological and ethical issues, microorganisms are considered as the best sources of proteases (Kelly and Fogarty 1976). Many past works have been done for the production of proteases by strains of Baccilus (Sattar et al. 2011), Rhizopus (Wang et al. 2006; Pandey et al. 2016) and Peniccillium (Djamel et

al. 2009; Haq et al. 2006). Fungi elaborate a wider variety of enzymes than bacteria. The fungal proteases 
are active over a wide $\mathrm{pH}$ range $(\mathrm{pH} 4.0-11.0)$ and exhibit broad substrate specificity. They are also used in the pharmaceuticals, medical diagnosis and in the detergent industry as additives (Joo et al. 2003). Fungal alkaline proteases are also used in food protein modification (Rao et al. 1998).

In present investigation a locally isolated strain Rhizopus oryzae (SN5) NCIM has been used for the production of proteases. In order to reduce the production cost of enzyme, various agro waste and low cost, readily available biomass has been used under solid state fermentation (SSF). Other important factors which impart great impact on the yield of enzyme are culture parameters such as temperature, $\mathrm{pH}$, inducers, carbon and nitrogen sources and their respective concentration. Therefore through various mathematical and computational optimization techniques, optimal combination of various culture parameters and substrate ratio have been achieved by researchers, such as one-variable-at-a-time, RSM [Milala et al. 2016], EVOP-factorial design technique [Negi and Banerjee, 2006; Pandey et al. 2016], Artificial Neural Network (ANN) [Román et al. 2011; Vats and Negi 2013], genetic algorithm etc. ANN has been preferred because of their proven advantages over other mathematical and computational method in order to get optimum physicochemical conditions for optimum yield of fermentation products (Yadav et al. 2013; Vats and Negi 2013). An artificial neuron network (ANN) is a computational model based on the structure and functions of biological neural networks and can be used to approximate any function that can depend on a large number of inputs. Neural networks are massively parallel and distributed processing systems representing a new computational technology built on the analogy to the human information processing system i.e. neural networks. Basic parts of ANN are neurons, which receives input from some other nodes or from an external source and computes an output. Each input has an associated weight $(\mathrm{w})$, which is assigned on the basis of its relative importance to other inputs, all neuron in network has a bias(b) associated with it. Three-layer Multilayer Feedforward Neural Network (MFNN) structure in which the input layer and the output layer are directly interconnected with the intermediate single hidden layer have the inherent capability to carry out any arbitrary input-output mapping with very high efficiency. When trained on examples of observation data, the networks can learn the characteristic features "hidden" in the examples of the collected data and even generalize the knowledge learnt. In present investigation ANN has been used for the optimization of the important physicochemical parameters such as combinational ration of different substrate as carbon source, $\mathrm{pH}$ and incubation temperature to achieve optimum yield of protease enzyme from locally isolated Rhizopus oryzae SN5.

\section{Materials And Methods}

\section{Chemicals}

The chemicals used in this study were all of analytical grade and all the substrates viz. wheat bran, rice husk, soybean meal and black gram husk were purchased from local market at Teliarganj, Allahabad211004, India.

\section{Maintenance of culture and inoculum preparation}


For the present work, Rhizopus oryzae (SN5)/NCIM-1447strain isolated from compost soil obtained from Ranikhet, Uttrakhand, India was utilized. This strain was characterised from NCIM, Pune India and deposited. Strain Seq286_NC111119 showed closest homology with Rhizopus sp. (closer to oryzae). This strain was maintained on Potato Dextrose Agar (PDA) slants at $4^{\circ} \mathrm{C}$. For preparation of inoculum, $25 \mathrm{~mL}$ of sterile distilled water was added to five days old slant and aseptically scraped with an inoculating loop. This suspension of spores was used as inoculum for the preparation of induced culture.

\section{Preparation of induced culture}

Medium for preparation of induced culture contained $1 \%$ casein, $0.25 \%$ glucose in modified Czapek-Dox broth. This medium was autoclaved and then inoculated with $2 \%$ of the spore suspension containing approximately $3.2 \times 10^{6}$ spores $/ \mathrm{ml}$. The medium was incubated at $28^{\circ} \mathrm{C}$ for 6 days. After the complete growth of the organism, the flask was placed on a shaker to make a homogenous suspension of spores.

\section{Fermentation set up}

The fermentation was carried out in $500 \mathrm{~mL}$ Erlenmeyer flasks, each carrying $5 \mathrm{gm}$ of different substrates viz. wheat bran, soybean meal, a mixture of wheat bran and soybean meal in ratio 4:1, black-gram husk and rice husk, respectively. The substrates were soaked in $10 \mathrm{~mL}$ of Czapek-Dox salt solution with pH 6 and autoclaved. This preparation was then inoculated with $1 \mathrm{~mL}$ of the induced spore suspension containing about $4.3 \times 10^{7}$ spores and kept in incubator at $28^{\circ} \mathrm{C}$. Fermentation was carried out in different experimental conditions changing one parameter at a time.

\section{Extraction of crude enzyme}

The fermented substrate was soaked in $50 \mathrm{~mL}$ of $0.1 \mathrm{M}$ phosphate buffer and kept on a shaker at $120 \mathrm{rpm}$ for 2 hours. The substrate was filtered through muslin cloth and the extract was centrifuged at $10,000 \mathrm{rpm}$ at $4^{\circ} \mathrm{C}$ for 10 minutes for removal of spores and other insoluble debris. The supernatant or crude enzyme extract was stored at $4^{\circ} \mathrm{C}$ for measurement of protease activity.

\section{Protease assay}

Protease activity was measured through modified caseinolytic method (Walter 1984). $0.65 \mathrm{~mL}$ of glycine$\mathrm{NaOH}$ buffer with pH 8 was incubated with $0.05 \mathrm{~mL}$ of enzyme solution for 10 minutes. $2 \mathrm{~mL}$ of $2 \%$ casein solution was then added and the reaction mixture was incubated at $37^{\circ} \mathrm{C}$ for $20 \mathrm{~min}$. The reaction was ceased by adding $0.1 \mathrm{~mL}$ of $1 \mathrm{M} \mathrm{HCl}$ and the non-hydrolysed casein was precipitated by $5 \mathrm{~mL}$ of $5 \%$ Trichloroacetic acid. The precipitated casein was removed through centrifugation at 10,000 rpm for 5 minutes. The protein concentration was measured in $0.5 \mathrm{~mL}$ of the supernatant through Lowry method using BSA as standard (Lowry et al. 1951). One unit of enzyme activity is defined as the amount of enzyme that liberates peptide fragments equivalent to $1 \mathrm{mg}$ of BSA per minute under the assay conditions.

\section{Effect of Process parameters on enzyme production}


Various process parameters such as temperature, carbon sources, incubation time, $\mathrm{pH}$, carbon and nitrogen sources, were studied to monitor their effect on alkaline protease production in Solid-State fermentation through one at a time first and then with EVOP factorial design technique.

\section{Effect of carbon sources}

$5 \mathrm{~g}$ each of different agro-industrial wastes such as wheat bran, rice husk, black-gram husk, soybean meal and a mixture of wheat bran and soybean meal in ratio 4:1 (in g) were used as substrate in different combinations for fermentation.

\section{Effect of incubation time}

The fermentation flasks were incubated for $24,48,72,96,120$ and 144 hours and protease activity was measured according to the procedure described earlier.

\section{Effect of changing ratio of Wheat Bran to Soybean Meal}

The ratio of Wheat Bran and Soybean Meal was changed as follows- 4.5:0.5, 4:1, 3:2, 2:3 (in g) and fermentation was done as described before.

\section{Effect of pH}

The initial pH of the substrate before autoclaving was maintained $4,5,6,7$ and 8 by changing the $\mathrm{pH}$ of Czapek-Dox broth using $1 \mathrm{~N} \mathrm{HCl} / 1 \mathrm{~N} \mathrm{NaOH}$.

\section{Effect of temperature}

Temperature was kept $28,30,35$ and $37^{\circ} \mathrm{C}$ by keeping the fermentation flasks in different incubators preset at respective temperature.

\section{Effect of nitrogen sources}

Czapek-Dox salt solution was supplemented with sodium nitrate (1\%), ammonium nitrate (1\%), peptone $(1 \%)$, yeast extract $(1 \%)$ and potassium nitrate $(1 \%)$ to study the impact of different nitrogen sources on alkaline protease production.

\section{Process parameters optimization through EVOP factorial Design technique}

The EVOP methodology was applied to determine the optimum levels of three parameters (ratio of wheat bran to soybean meal, $\mathrm{pH}$ and temperature) in different experiments. Firstly, the control experimental conditions ( $A 1$ and $A 6$ ) were selected based on the results obtained from one factor at a time optimization. Then, new experimental conditions with higher and lower search levels of parameters were selected. The experimental design for three variables system is shown in Table 1. Thirdly, fermentation was carried out as described earlier at higher and lower search levels and all the experiments were repeated for two cycles. The yield of alkaline protease in cycle I and cycle II was measured following the procedure mentioned earlier. The differences in protease yield in cycle I and cycle II were calculated along 
with the averages in order to estimate the effects and error limits. The magnitude of the effects, error limits and changes in the main effect were inspected as per the decision making procedure to arrive at the optimum.

\section{Decision making}

Once all the calculations for effects and error limits are completed, it is extremely necessary to determine whether any changes in the control level will help to enhance the objective function and if the changes are required then the next step is to identify the desired direction of change. For this study, magnitudes of the effects were compared to those of the error limits. If all or any of the effects are higher than the error limits, alterations in the experimental conditions may yield better results.

\section{Optimization of process parameters through ANN Method}

Data selected for optimization

Data used in ANN was output of Evop-Factorial design technique and one factor at a time i.e., $\mathrm{pH}$, incubation temperature and different percentage of two different substrate combination of Wheat bran(WB)and Soybean mil(SM) [Table 2].

The data selected for optimization was used to fit a nonlinear regression:

\section{Protease activity $=-2299.6275+7.896875^{\star} x(1)-0.591625^{\star} x(2)+839.270625^{\star} x(3)-69.7646875^{\star} x(3)^{\wedge} 2$}

Where $: x(1)=$ temperature value,$x(2)=W B$ percentage in the WB and SM substrate mixture,

$$
x(3)=p H \text { value }
$$

Optimization methodology: This analysis was carried out in MATLAB, Windows 8 operating system, Intel R CPU $2.53 \mathrm{GHz}, 8.00 \mathrm{~GB}$ of RAM. The input data and target data was the data collected from the Table 2, which was fed into the designed neural network for training. The speed of this process varies according to the specifications of the system. There are different types of neural network available for training and optimization. However, we had chosen to solve this particular problem using feed forward network and trained by back propagation algorithm. The network flow is only in one direction. There was no feedback because we wanted to create a simple network for this problem. This network used supervised learning so input and output data were fed to the network. In case of unsupervised learning, only input has given and linear equations were built such that maximum correlation coefficient was achieved. Signals flow forward and errors are propagated backward to ensure that errors were reduced and $\mathrm{R}$ square value increased closer to 1 . Random weights were initialized and changed in each run of each iteration to made change in learning process. The objective was to minimize the errors. The different parameters 
considered in the neural network included training functions, performance measures, number of layers, number of hidden layers and neurons, and transfer function.

Network Architecture and Training of MFNN: A three layer (3-10-1) MFNN was designed using MATLAB: 1 input layer (3 neurons), 1 hidden layer(10),1 output layer(1 neuron). Layers were kept fully connected means each neuron in next layer has been connected to each neuron in previous layer. The sigmoidal function was used as transfer function of neurons in hidden layer and linear function was used as transfer function of output layer. Sigmoidal function gives more accuracy with backpropagation algorithm. 'Trainlm' was used for training the MFNN because it is a fast and stable algorithm[M63]. The network was trained for 64 times to find best fit to the data. The training results of The R square value was found 0.9967 for overall, 0.99909 for Training, 0.9994 for validation and 0.988 for text, which is very close to one and acceptable for good fitting. Performance of MFNN is shown in Figure (1a) and Figure (1b).

Calculating network output: The trained neural network was used to generate protease activity at various input values of temperature, $\mathrm{WB} \%$, and $\mathrm{pH}$ value setting constraints.

(1) $26<=$ temperature $<=30$ at increment of $1^{\circ} \mathrm{C}$

(2) $70<=W B \%<=90$ at increment of 5

(3) $4<=\mathrm{pH}<=8$ at increment of 0.2 .

Network output was calculated at 1850 possible combinations of inputs to achieve our goal of optimization of protease activity. The network output has displayed in Figure (2a) and (2b). Individual combination of temperature, $\mathrm{pH}$ and $\mathrm{WB} \%$ was given an iteration number which was plotted in $\mathrm{x}$-axis and the corresponding protease activity of network output was plotted in $\mathrm{y}$-axis as shown in the Figure (2a).

The maximum protease activity was between $1000^{\text {th }}$ to $1100^{\text {th }}$ iterations. The network response of between these values was further plotted in the Figure (2b). It was found again that maximum protease activity was in between $1020^{\text {th }}$ to $1030^{\text {th }}$ iteration. The network response for these values is shown in the Table 4.

\section{Results}

The present study showed that out of the five substrates-wheat bran, rice husk, soybean meal, black gram husk and mixture of wheat bran and soybean meal; maximum alkaline protease production was observed by using mixture of wheat bran and soybean meal. The optimum ratio of wheat bran and soybean meal was found to be 4:1 (in g). The optimization of physiological parameters was initially done with one 'factor at time method'. First incubation period was varied keeping other factors constant and maximum protease activity was observed after 72 hours of incubation. A rapid reduction in protease activity was observed when the incubation temperature was raised beyond $28^{\circ} \mathrm{C}$. Rhizopus oryzae SN-5 strain secreted maximum amount of alkaline proteases at $\mathrm{pH}=6$. The nitrogen requirement of the strain for maximum secretion of proteases was found to be satiated by $1 \%$ sodium nitrate. 
Based on the above findings, ratio of wheat bran to soybean meal, $\mathrm{pH}$ and temperature were selected as the critical process parameters and their optimum values were taken as initial search level in EVOP factorial design technique. Following the procedure, as described earlier, the design of three variables system has given in Table 1. The results of phase I of investigation have been shown in Table 2 where A1 and $A 6$ represent the initial optimum conditions for the three parameters for the maximum production of alkaline protease. For the new sets of experiments, each variable parameter possessed two levels of magnitude, one lower and one higher, which were as follows: temperature $(28 \pm 2){ }^{\circ} \mathrm{C}, \mathrm{pH}(6 \pm 2)$ and ratio of wheat bran (WB) to soybean meal (SM) (4:1 \pm 0.5$)$. As shown in Table 3 , the calculations of the effects and analysis of the results of cycle I and cycle II for protease production showed that the changes in the main effect were larger and negative $(-231.94,-214.54)$ and all the effects were smaller than the error limits $( \pm 101.04, \pm 93.40)$. The maximum production of protease was obtained at initial optimum conditions i.e A6. From this analysis, it was concluded that optimum conditions for the production of alkaline proteases have been achieved. The yield of alkaline protease was $412.79 \mathrm{U} / g d s$ under the optimized conditions.

Further data obtained in EVOP has been considered as input data in ANN and used for training of software also. On processing the data in $\mathrm{ANN}$, optimum conditions of $\mathrm{pH}$, temperature and substrate that accounts for maximum protease activity of $422.66 \mathrm{U} / \mathrm{ml}$ were: temperature- $30^{\circ} \mathrm{C}$, optimum WB substrate percentage in a mixture of WB- $70 \%$ and optimum pH-6.2. The Levenberg-Marquardt algorithm solves the problems existing in both gradient descent method and the Gauss-Newton method for neuralnetworks training, by the combination of these two algorithms. The protease activity was found increased about 1.5 times from the experimental results. The validation result was also found fit to the result of ANN. The result of ANN and EVOP matches statistically. The results of EVOP technique are as shown in Table 2 and Table 3.

Yield of alkaline protease was $412.79 \mathrm{U} / \mathrm{gds}$ under the optimized conditions obtained through EVOP technique and $422.66 \mathrm{U} / \mathrm{ml}$ with ANN optimization methodology, which is higher than EVOP.

\section{Discussion}

The aim of present study was to optimize physiological conditions for maximum production of alkaline proteases from cost-effective and readily available substrates. Wheat bran and soybean meal both are agro industrial wastes that are low-cost and highly ubiquitous. During optimization studies, it was observed that fermentation carried out using a mixture of wheat bran and soybean meal produced maximum protease as compared to when wheat bran and soybean meal were used alone. The texture, porosity and carbohydrate content of wheat bran supports the growth of the organism, however, addition of protein rich soybean meal enhanced the protease production by $17 \%$. While optimizing the ratio of wheat bran to soybean meal to be used in fermentation, it was observed that protease production decreased with the increase in the fraction of soybean meal. This observation may be explained by the presence of a number of protease inhibitors in soybean. Wheat bran and soybean meal have been used as substrates individually by various researchers for production of proteases (Chandran et al. 2005; Qazi 
et al 2008). Agrawal et al.(2004) obtained good protease activity from Penicillium sp. using wheat bran supplemented with $25 \mathrm{mg}$ of soy protein per gram wheat bran. In another study conducted by Qazi et al. (2008) a mixture of wheat bran and soybean in ratio 8:2 (in g) was used for enhanced production of proteases using Aspergillus niger. Alkaline protease production by Rhizopus sp. utilizing a mixture of wheat bran and soybean has not been explored earlier.

Protease activity was increased drastically by inducing the organism prior to fermentation. This is done merely to reduce the lag phase of the organism. It was found that induction of the strain by using $1 \%$ casein and $0.5 \%$ glucose increased the protease activity by 2.14 -fold. This is the most economical and effortless method for induction of the strain to improve the yield of enzyme, without deteriorating the quality of the product as well as that of the organism.

Alkaline protease activity was highest after 72 hours of incubation. A rapid decline in protease activity was observed after 96 hours. Protease degradation after maximum enzyme production is well known in literature (Chu et al. 1992; Prasad et al. 1995). The accurate mechanism underlying this type of reduction in protease synthesis is not properly understood. However, autoproteolysis and high vulnerability of proteases to various inhibitors are hypothesized to be the major reasons behind the reduction of protease activity.

Temperature and $\mathrm{pH}$ play a pivotal role in production of extracellular enzymes. Alkaline protease production was found to be highest at $30^{\circ} \mathrm{C}$ for the growth of this strain. At $\mathrm{pH}=6.2$, the secretion of protease was maximum, this suggests that the organism is able to produce proteases in slightly acidic conditions. These physiological conditions are ideal for the utility of this strain in protease production for applicability in detergent, leather and food-processing industries.

In order to achieve optimum physicochemical conditions for maximum yield of protease, three approaches: one factor at time, EVOP and ANN were assessed in current study. Yield of protease was highest $422.6 \mathrm{U} / \mathrm{gds}$ at physicochemical conditions obtained through ANN, because ANN method enables determination of interaction effects and error limits by making a small variation around the initial optimum conditions, which is not possible in other methods. ANN is simple, time-effective and highly reliable method as it does not require alteration in the existing plant and also poses no risk to product's quality. Negi and Banerjee (2006) optimized parameters such as temperature, $\mathrm{pH}$ and relative humidity for the concomitant production of protease and amylase from Aspergillus awamori through EVOP methodology.

A number of reports are available on alkaline proteases production from microbial sources. But, microorganisms with superior growth kinetics and inexpensive medium requirements producing highly stable proteases in short amount of time (within 72 hours) are not well-known using agro industrial wastes such as wheat bran and soybean meal. In the current comparative study, ANN methodology was found better and cheaper way of optimization methodology than EVOP method. Yield of protease was found maximum at optimum physicochemical conditions obtained through ANN. 
This work has demonstrated the use of ANN to optimize the culture condition of Rhizopus oryzae for the optimum yield of alkaline protease. The experimental design of ANN maximizes the amount of information that can be obtained, while reducing the number of individual experiments needed to be performed. It is less-time consuming and can be utilized for optimization of various fermentation processes. This study confirms the immense potential of Rhizopus oryzae SN-5 strain to produce large amount of alkaline proteases at low cost in comparatively shorter time.

\section{Declarations}

\section{Ethics approval and consent to participate}

Current research report doesn't contain any data collected from humans or animals and also there was no involvement of human participants and doesn't belongs to any health related outcomes and reports.

\section{Consent for publication}

This research article entitled as "Combined ANN/ EVOP factorial design approach for media screening for cost-effective production of Alkaline proteases from Rhizopus Oryzae SN-5 under SSF " an original work was carried out by authors: Sangeeta Negi and Sapna Jain and Anand kumar, who all are agree to submit this Manuscript to AMB express journal for probable publication. The manuscript has not been previously published, is not currently submitted for review to any other journal, and will not be submitted elsewhere before a decision is made by this journal.

I am the author responsible for the submission of this article and I accept the conditions of submission and the Springer Open Copyright and License Agreement.

\section{Availability of data and material}

It is the responsibility of each author for providing authenticated data and material and data would be made available also.

\section{Competing interests}

As a corresponding author, I confirm that I have read Springer Open's guidance on competing interests and have included a statement indicating that none of the authors have any competing interests in the manuscript.

\section{Funding}

The authors are grateful to Technical Education Quality Improvement Programme (TEQIP), India (Institute internal funding) for providing partial financial assistance for the present work.

\section{Authors' contributions}


Current work has been performed by Miss Sapna Jain during her M.Tech project/thesis work under the supervision of Dr. Sangeeta Negi (corresponding author) and Anand Raj was our B. Tech student who helped in implementing ANN methodology for process optimization.

\section{Acknowledgements}

The authors acknowledge to Department of Biotechnology, MNNIT Allahabad for providing lab facility to carry out this work.

\section{Authors' information (optional)}

Corresponding author is working as an Associate Professor as permanent employ of MNNIT

ALLAHABAD, Miss Sapna Jain was M.Tech (pass out) student and Mr Anand Raj was B.Tech student.

\section{References}

Agrawal D, Patidar P, Banerjee T, Patil S (2004) Production of alkaline protease by Penicillium sp. under SSF conditions and its application to soy protein hydrolysis. Process Biochem. 39:977-981.

Chandran S, Alagarsamy S, Szakacs G, Pandey A (2005) Comparative evaluation of neutral protease production by Aspergillus oryzae in submerged and solid-state fermentation. Process Biochem. 40:2689-2694.

Chu IM, Lee C, Li TS (1992) Production and degradation of alkaline protease in batch cultures of Bacillus subtilis ATCC 14416. Enzyme Microb Technol. 14:755-61.

Djamel C, Ali T, Nelly C (2009) Acid protease production by isolated species of Penicillium. Eur J Sci. Res. 25:469-477.

Ezendam J., Opperhuizen A., Loveren van H. (2006) Immunomodulation by probiotics: efficacy and safety evaluation. RIVM report 340320003/2005.

Haq IU, Mukhtar H, Umber H (2006) Production of protease by Penicillium chrysogenum through Optimization of Environmental Conditions. J Agri Soc Sci. 2:23-25.

Joo HS, Kumar CG, Park GC, Paik SR, Chang CS (2003) Oxidant and SDS-stable alkaline protease from Bacillus clausii I-52: Production and some properties. J Appl Microbiol. 95: 267-272.

Kelly CT, Fogarty WM (1976) Microbial alkaline enzymes. Process Biochem. 11:3-9.

Lebeer S, Vanderleyden J, De Keersmaecker SC (2010) Host interactions of probiotic bacterial surface molecules: comparison with commensals and pathogens. Nat. Rev. Microbiol. 8(3):17184. doi:10.1038/nrmicro2297. 
Lowry OH, Rosenbrough NJ, Farr AL, Randall RJ (1951) Protein estimation with the Folin-phenol reagent, J. Biol. Chem. 193:265-275.

Milala MA, Jatau IA and Abdulrahman AA (2016) Production and Optimization of Protease from Aspergillus niger and Bacillus subtilis using Response Surface Methodology. J. Biotechnol and Biochem. 2(7): 01-07.

Negi S, Banerjee R (2006) Optimization of Amylase and Protease Production from Aspergillus awamori in Single Bioreactor through EVOP Factorial Design Technique. Food Technol. Biotechnol. 44(2):257261.

Pandey AK, Edgard G, Negi S (2016) Optimization of concomitant production of cellulase and xylanase from Rhizopus oryzae SN5 through EVOP-factorial design technique and application in Sorghum Stover based bioethanol production, Renewable Energy.98:51-56.

Prasad S, Banerjee R, Bhattacharyya BC (1995) Production of proteolytic enzyme in a bubble column bioreactor by Rhizopus oryzae. J Bioprocess Eng. 13:41- 43.

Protein Hydrolysis Enzymes Market by Sources (Microorganisms, Animals, Plants), Applications (Detergent Industry, Pharmaceuticals, Food Industry and Others), \& Geography (North America, Europe, Asia-Pacific \& ROW) - Global Trends \& Forecasts to 2019.

Qazi Jl, Jamshaid H, Nadeem M, Ali SS (2008) Production of proteases by Aspergillus niger through solid state fermentation. Punjab Univ J Zool. 23(1-2): 037-046.

Rao MB, Tanksale AM, Ghatge MS, Deshpande VV (1998) Molecular and biotechnological aspects of microbial proteases. Microbiol Mol Biol Rev. 62: 597-635.

Román RC, Hernández OG, Urtubia UA (2011) Prediction of problematic wine fermentations using artificial neural networks. Biopro and Biosys Eng 34(9):1057-1065.

Sattar AQ, Aqeel MB, Imrana K, Umar MD (2011) Optimization of Cultural Conditions for Protease Production by Bacillus subtilis EFRL 01. Afr J Biotechnol.10 (26): 5173-5181.

Vats S, Negi S (2013) Use of artificial neural network (ANN) for the development of bioprocess using Pinus roxburghii fallen foliages for the release of polyphenols and reducing sugars. Bioresour Technol. 140:392-398.

Walter HE, (1984) Proteinases: Methods with Hemoglobin, Casein and Azocoll as Substrates. In: Methods of Enzymatic Analysis, Vol. 5, (Bergmeyer HU, Bermeyer J Eds.), John Wiley \& Sons Ltd., New York, USA, pp. 270-274.

Wang HY, Liu DM, Liu Y, Cheng CF, Ma QY, Huang Q, Zhang YZ (2006) Screening and Mutagenesis of A novel Bacillus pumilus Strain Producing Alkaline Protease for Dehairing. Lett. Appl. Microbiol. 44:1-6. 
Yadav M, Sehrawat N, Sangwan A, Kumar S, Beniwal V, Singh AK (2013) Artificial Neural Network (ANN): Application in media optimization for industrial microbiology and comparison with response surface methodology (RSM). Adv Appl Sci Res. 4(4):457-460.

\section{Tables}

Due to technical limitations, the tables are only available as a download in the supplemental files section.

\section{Figures}



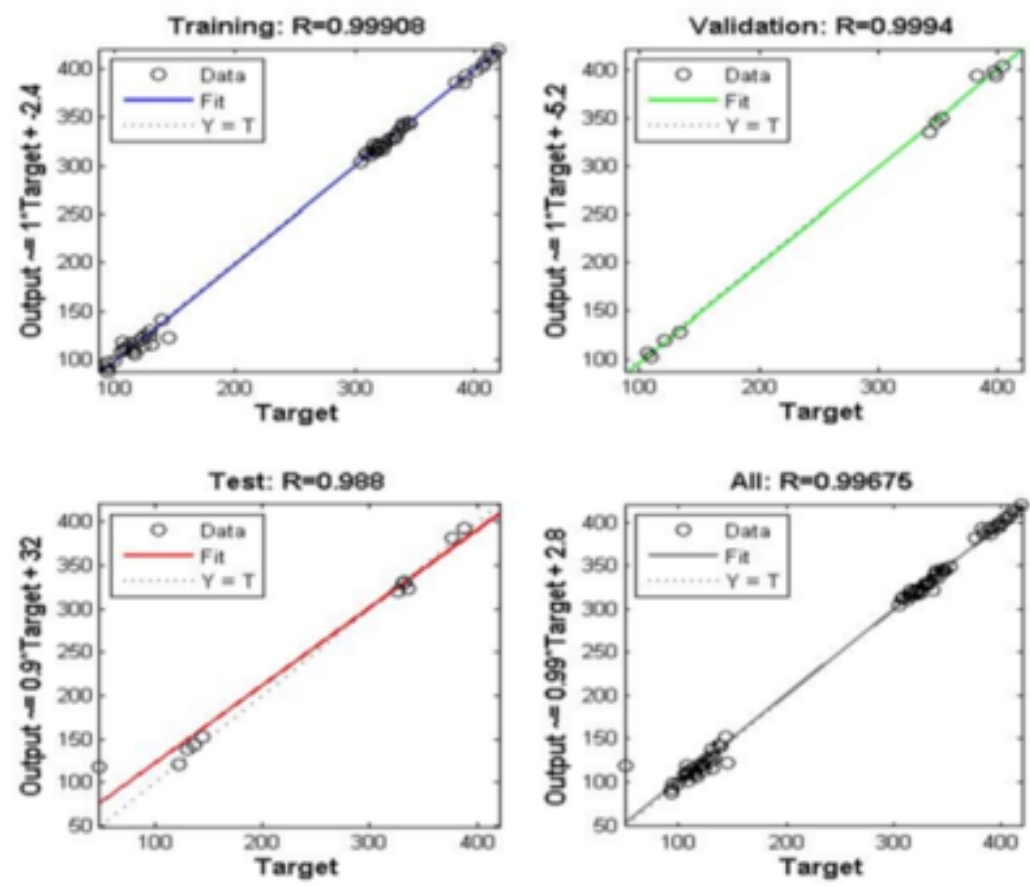

Fig(1a). The graphical depiction of the regression results of training, validation and testing

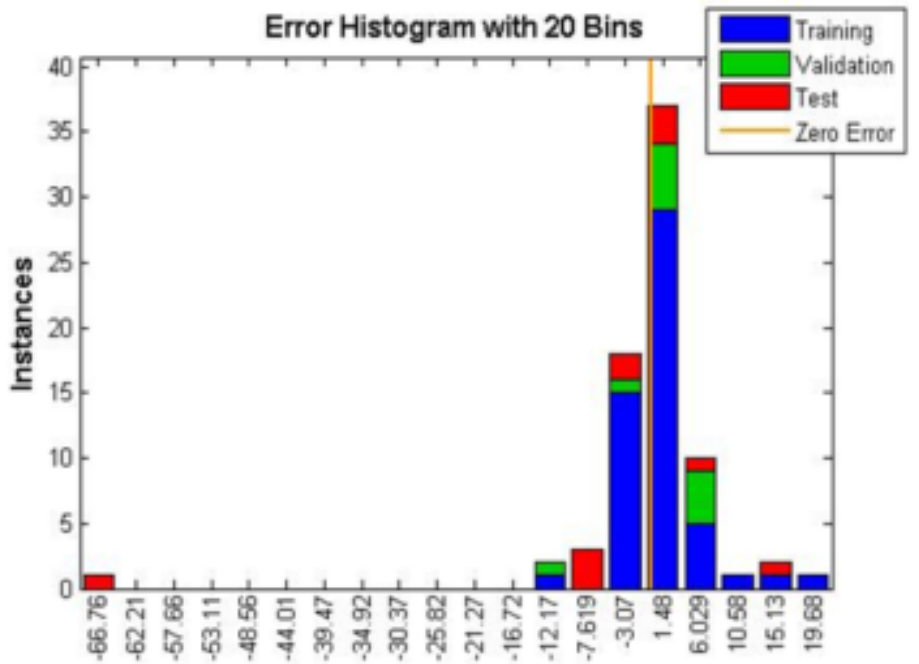

Errors $=$ Targets - Outputs

Figure(1b):Error histogram plot of training of Network

\section{Figure 1}

(a). The graphical depiction of the regression results of training, validation and testing (b):Error histogram plot of training of Network 


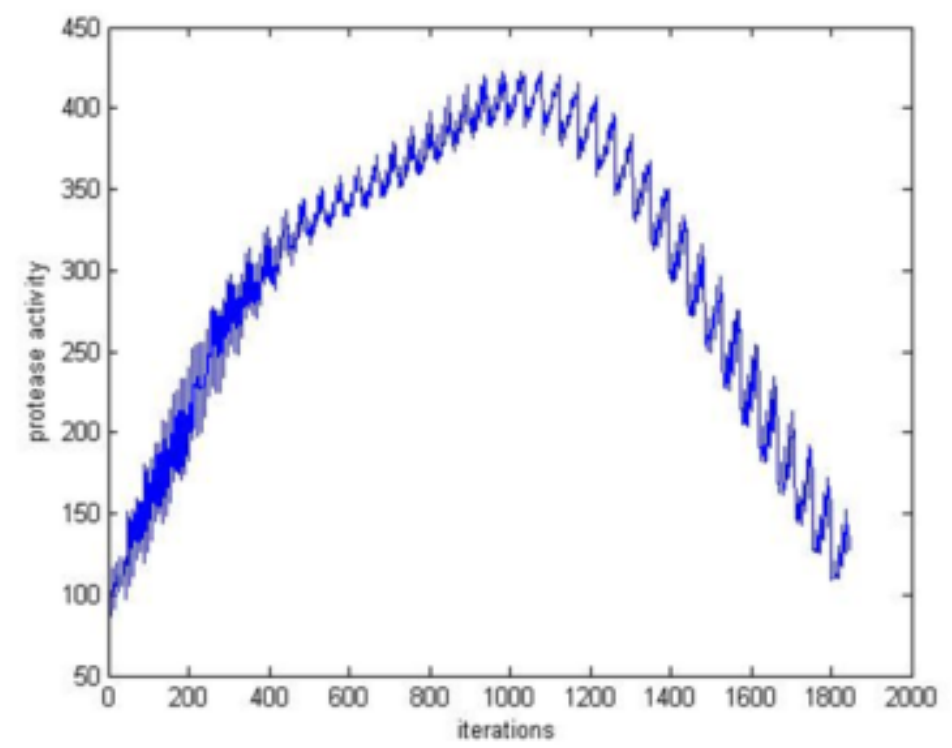

Figure (2a): Predicted protease activity response of the trained ANN network.

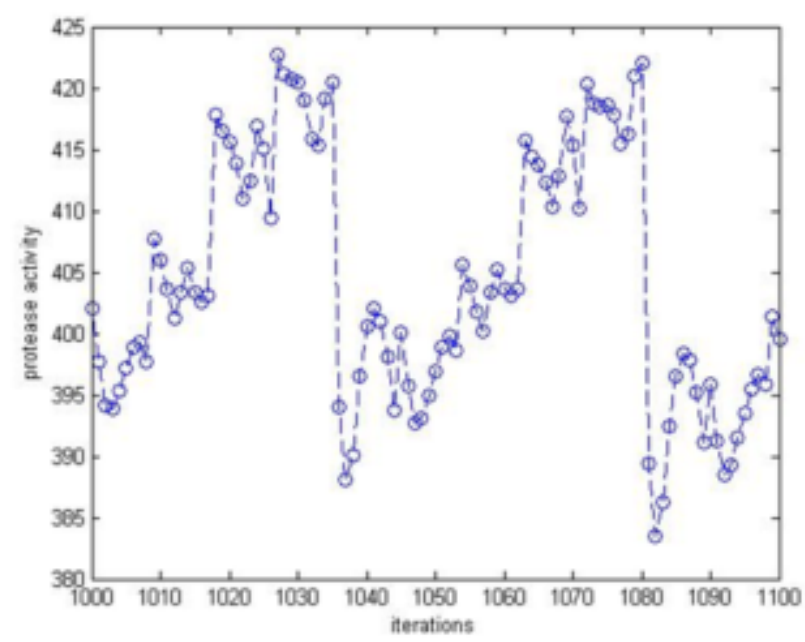

Figure (2b): Region of maximum network response

Figure 2

(a): Predicted protease activity response of the trained ANN network. (b): Region of maximum network response

\section{Supplementary Files}

This is a list of supplementary files associated with this preprint. Click to download.

- Tables.pdf 
Page 16/16 